\title{
A MOBILE EEG STUDY ON THE NEUROPHYSIOLOGICAL CORRELATES OF ORAL READING IN DYSLEXIA
}

\author{
Katherine Ko ${ }^{1}$, Roann $\operatorname{Ramos}^{2,3}$, \& Rosalito De Guzman ${ }^{4}$ \\ ${ }^{1}$ Department of Psychology, College of Science, University of Santo Tomas (Philippines) \\ ${ }^{2}$ RWTH Aachen University Hospital (Germany) \\ ${ }^{3}$ College of Education Graduate Studies, De La Salle University-Dasmariñas (Philippines) \\ ${ }^{4}$ The Graduate School, University of Santo Tomas (Philippines)
}

\begin{abstract}
Cognitive neuroscientists have already begun to examine the neural basis of reading in dyslexia. In this study, the neurophysiological characteristics of reading in Filipino children with dyslexia were investigated. Using mobile EEG (mEEG), the researchers sought to determine brain differences between four normal reading children (NR group; mean age $=9.61 ; \mathrm{SD}=1.00)$ and five children with dyslexia (DYS group; mean age $=9.61 ; \mathrm{SD}=1.7$ ) as they were tasked to read regular words, irregular words, and non-words. Significant differences were revealed for irregular words $(\mathrm{U}=0, \mathrm{p}=.01)$ and non-words $(\mathrm{U}=1.5, \mathrm{p}=.04)$, but not for regular words $(\mathrm{U}=3, \mathrm{p}=.08)$. $\mathrm{mEEG}$ analysis revealed that the DYS group exhibited slower brain activity in the left frontal and posterior regions, as well as stronger activity in the right hemisphere. These findings signify that the reading impairment in dyslexia is caused by reduced communication and weaker connectivity in the frontal and posterior regions which are important areas for visual word processing. Furthermore, stronger connectivity in the right hemisphere during reading indicates a compensatory mechanism in dealing with reading difficulties.
\end{abstract}

Keywords: Dyslexia, reading, mobile EEG.

\section{Introduction}

Dyslexia is a neurodevelopmental disorder characterized by severe and persistent reading difficulties despite normal intellectual functioning and appropriate schooling (Pina Rodrigues et al., 2017). Although dyslexia is usually only diagnosed after two to three years of schooling, neuroanatomical precursors have been identified in pre-reading children at family risk for the disorder (Goswami, Power, Lallier, \& Facoetti, 2014; Clark et al., 2014). This implies that if a child has a parent or a sibling with dyslexia, it is important that he or she be carefully observed for signs of reading difficulties. One approach to understanding reading is through the dual-route cascaded (DRC) model of visual word recognition and reading aloud by Coltheart and colleages (2001). The DRC model proposes that written language is processed by two independent but interactive pathways: the lexical route and the sub-lexical route. The lexical (or direct) route processes familiar and orthographically irregular words but would fail to process unfamiliar words or pseudowords. The sub-lexical (or indirect) route, on the other hand, involves an understanding of grapheme-to-phoneme correspondences through which one can read unfamiliar words and pseudowords but fail to produce accurate responses to irregular words. To be a skilled reader, one must acquire both these routes. On the other hand, an impaired reader is characterized by a dysfunction in either or both of these routes (Bosse \& Valdois, 2009; Gori, Cecchini, Bigoni, Molteni, \& Facoetti, 2014).

Reading requires the cooperation and integration of several neurocognitive processes situated in multiple brain regions. According to the neural model of reading by Shaywitz and Shaywitz (2008), a number of interrelated neural systems, primarily located in the left hemisphere, are involved in reading: 1) the dorsal system, which involves phonologically-based reading and facilitates grapheme-phoneme conversions, includes the posterior superior temporal and inferior parietal regions; 2) the ventral system, which consists of left ventral occipito-temporal areas and is associated with visual-orthographic word recognition; and the 3) the anterior system, which involves the left inferior frontal region and facilitates speech articulation and word analysis (Martin, Kronbichler, \& Richlan, 2016; Richlan, 2014). To investigate the neural dynamics of reading, researchers in the field of cognitive neuroscience use neuroimaging techniques such as mobile electroencephalography (mobile EEG or mEEG), which allows researchers to observe patterns of brain frequencies (or waves) in a practical, accessible, and easy-to-use manner (Badcock et al., 2015). Each frequency band underlying function: A dominance of slow 
frequencies (i.e., delta and theta) when one is engaged in a cognitive task would suggest slow brain activity and possibly even cognitive dysfunction (Kamel \& Saeed Malik, 2015), whereas faster frequencies (i.e., beta and gamma) are dominant when the brain is actively processing information (Magazzini \& Singh, 2018). The alpha band, which is dominant at resting state, is associated with cortical and behavioral inhibition (Bastos et al., 2015). These frequencies are used to explain the differences in brain activation between normal controls and those with neurological deficits. In normal readers, stronger activity characterized by a dominance of beta and gamma frequencies is typically found in the left hemisphere. In constrast, brain activation in readers with dyslexia typically involve a dominance of slow frequencies (i.e., delta and theta) in the left hemisphere, reflecting a dysfunction in the left hemisphere reading network (González-Garrido, Gómez-Velázquez, \& Rodríguez-Santillán, 2014; Jiménez-Bravo, Marrero, \& Benítez-Burraco, 2017; Paulesu, Danelli, \& Berlingeri, 2014; Richlan, Kronbichler, \& Wimmer, 2013; Shaul, Arzouan, \& Goldstein, 2012; Žarić et al., 2014, 2017). The aim of this study is to illustrate the neurophysiological characteristics of Filipino children with dyslexia as they engage in an oral reading task using $\mathrm{mEEG}$ as a brain imaging tool.

\section{Method}

\subsection{Participants}

The participants were divided into the Dyslexia (DYS) group $(\mathrm{n}=5$; mean age $=9.61 ; \mathrm{SD}=1.7)$ and the Normal Reader $(\mathrm{NR})$ group $(\mathrm{n}=4$; mean age $=9.61$; $\mathrm{SD}=1.00)$. For both groups, non-verbal IQ results obtained using the Raven's Colored Matrices (Raven, Raven, \& Court, 2003) were found to be within the normal range (at least at 75th percentile or an IQ of at least 90). The DYS group had been previously diagnosed with Specific Learning Disorder with an impairment in reading by a professional (medical doctor, clinical/educational psychologist, or special educator/reading specialist). On the other hand, the NR group had no history of reading difficulties. All participants were male, right-handed, with normal vision (as previously assessed by their physicians), and free of any co-morbid conditions, such as attention deficit/hyperactivity disorder, autism spectrum disorders, and any speech/language and visual impairments. Furthermore, parental consent and child assent were obtained before data gathering.

\subsection{Experimental task}

The Castles and Coltheart Reading Test 2 (CC2; Castles, Coltheart, Larsen, \& McArthur, 2009) was used to assess the functioning of the key processes in single word reading: sounding-out ability and whole word recognition ability. Sounding-out ability involves knowledge about letter-sound relationships and is assessed by accuracy in reading aloud nonwords (e.g., /gop/). Whole word recognition ability involves knowledge about familiar words and is assessed by accuracy in reading aloud irregular words (e.g., /yacht/), which cannot be read correctly via sounding-out rules. The test also includes regular words, such as /cat/, which can be read correctly via either sounding-out or whole word recognition processes. Consisting of 40 regular words, 40 irregular words, and 40 nonwords, test items were presented one-at-a-time, in mixed order, and with gradually increasing difficulty. Presentation was discontinued on each subscale after five consecutive items are read incorrectly. This test is administered to children ages six years to 11 years and 6 months. For each list, the total accuracy score was obtained and converted to into a z-score.

Figure 1. Illustration of the Oral Reading Task (CC2).

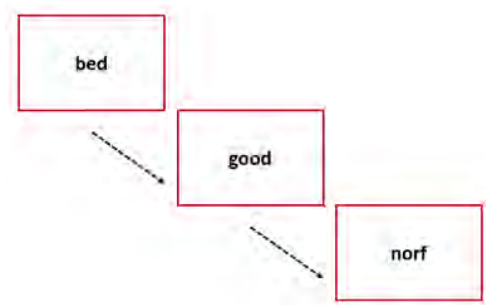

In this study, the online version of CC2 was used. The participants would sit in front of the computer screen as the instructions were read to them. Once it was clear that they understood what to do, the experimenter would click on "RUN TEST." The experimenter would mark their first response using the appropriate key and the next word would be prompted. If the child would not respond to a word within five to 10 seconds, he/she would be prompted to guess. If the child would be unable to respond within another five seconds, he/she was assured that it was alright and the incorrect response key was pressed. The program stopped automatically when the participants reached their limit of ability (Macquarie Online Test Interface, 2009). 


\subsection{Data collection and analysis}

Brain signals were be obtained by the Emotiv EPOC Neuroheadset (Emotiv Systems, Inc., 2013), a non-invasive, high-resolution, neuro-signal acquisition and processing wireless headset designed for contextualized research (see Figure 1). It has 14 channels (AF3, F7, F3, FC5, T7, P7, O1, O2, P8, T8, FC6, F4, F8, and AF42) distributed according to the internationally accepted 10-20 system of electrode placement and includes two references in the CMS/DRL noise cancellation configuration P3/P4 locations. Only 12 channels were included in the study (i.e., T7 and T8 were excluded).

Figure 2. The Emotiv EPOC Neuroheadset and its scalp locations.

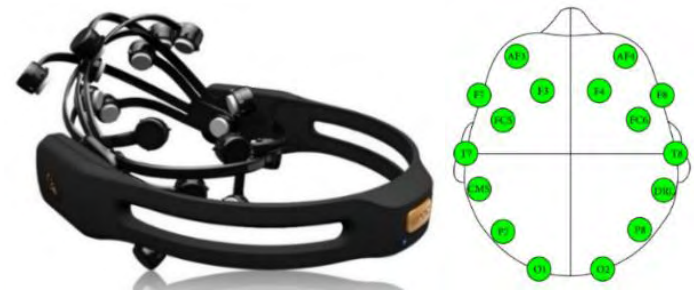

Data were transferred via Bluetooth to the computer and raw EEG data were acquired using the EmotivPRO software. Further signal processing was carried out using EEGLAB, an open source MATLAB toolbox for processing data from EEG. The EEG recordings were segmented into epochs to be extracted, visually inspected, and cleaned for artifacts. Absolute power analyses using fast Fourier transform (FFT) for delta $(1-4 \mathrm{~Hz})$, theta $(4-8 \mathrm{~Hz})$, alpha $(8-12 \mathrm{~Hz})$, and beta $(12-25 \mathrm{~Hz})$. Mann-Whitney U tests were performed using IBM SPSS Statistics version 25.0. Before putting on the headset, the 14 electrode recesses were fitted with a moist felt pad. The headset was then placed on the participant's head and subjected to software set-up which took approximately 10 minutes. After verifying that the built-in battery was fully charged and the wireless signal reception was reported as good, the experiment began. The participants were instructed to relax and keep their eyes closed for five minutes.

\section{Results and discussion}

\subsection{Oral reading task}

In order to determine significant group differences in reading accuracy assessed by the oral reading task, Mann-Whitney U tests were carried out. As seen in Table 1, the performance of the NR group was significantly higher than the performance of the DYS group on two reading lists: irregular words $(U=0, p=.01)$ and non-words $(U=1.5, p=.04)$. No significant difference was revealed for regular words $(\mathrm{U}=3, \mathrm{p}=.08)$. These results suggest that the DYS group suffer from an impairment in both lexical and sub-lexical reading wherein they not only exhibit poor word knowledge and recognition of unusual words, but also demonstrate ineffective grapheme-phoneme correspondences and difficulties in both visual and phonological encoding. The most severe reading deificits involve a dysfunction in both irregular word and non-word reading (Bosse \& Valdois, 2009; Gori et al., 2014).

Table 1. Mean ranks and sum of ranks for regular word, irregular word, and non-words in the oral reading task.

\begin{tabular}{lccccc}
\hline & NR & Group & & & \\
\cline { 2 - 4 } Regular Word & 6.75 & DYS & & p-value \\
Irregular Word & $(27)$ & $(18)$ & 3 & .08 \\
& 7.50 & 3.00 & & $0 *$ & .01 \\
Non-word & $(30)$ & $(15)$ & & \\
& 7.13 & 3.30 & $1.5^{*}$ & .04 \\
\hline
\end{tabular}

\section{2. mEEG analysis}

For the DYS group, delta and theta power was concentrated in frontal and parietal regions, signifying slower brain activity in these areas. Alpha power was concentrated in the frontal and right parietal regions, whereas beta power was exhibited at the frontal and occipital regions. On the other hand, gamma power activation was significantly stronger for the NR group. The DYS group was found to have a right-lateralized activation network, as well as increased delta power that is lateralized to the right hemisphere. 
Figure 3. Power distributions according to frequency band for Resting State. Power (in dB) is represented by colors $($ dark red $=$ very high, orange $=$ high, yellow $=$ average, light blue $=$ low, dark blue $=$ very low $)$.

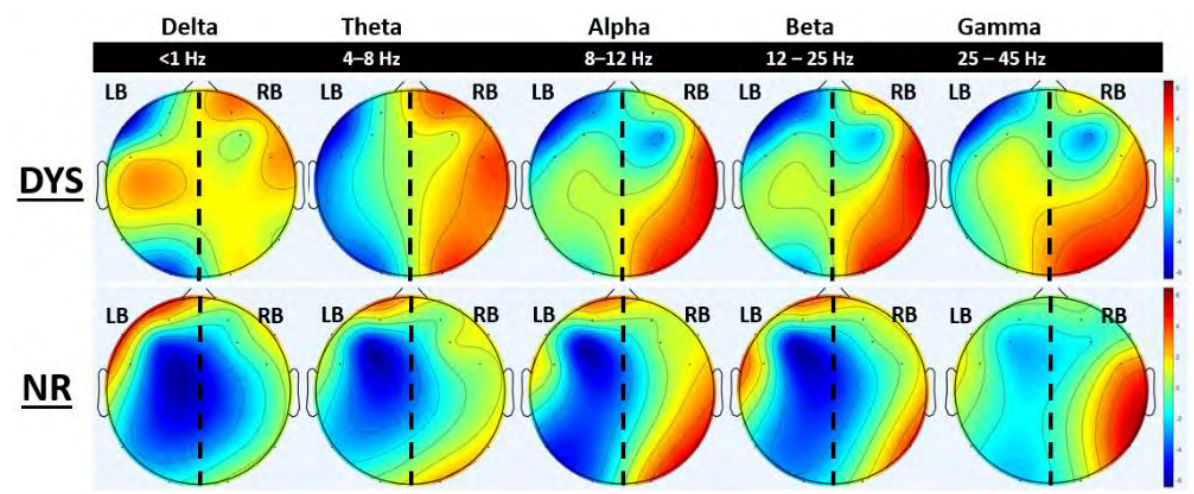

The results of the mEEG experiment show that the reading impairment in dyslexia is reflective of reduced communication and weaker connectivity in the left frontal and posterior regions, which are all involved in the reading and visual-to-phonology processes (Paulesu et al., 2014). Moreover, the DYS group demonstrated increased right hemisphere activity. Previous studies have also reported evidence of right-lateralized hyperactivations in dyslexia while reading, indicating a dysfunction of a left hemisphere reading network consisting of areas involved in speech processing and reading acquisition (Jiménez-Bravo, Marrero, \& Benítez-Burraco, 2017; Richlan, Kronbichler, \& Wimmer, 2013). Overall, these findings suggest a compensatory mechanism involving the right posterior brain regions when a disruption in visual word processing occurs (González-Garrido et al., 2014; Shaul et al., 2012; Žarić et al., 2014, 2017).

\section{Conclusions}

Using mEEG as a brain imaging tool, this study confirmed the existence of an atypical left hemipshere reading network marked by a dominance of slow frequencies in left cortical areas, as well as a possible compensatory mechanism for reading in dyslexia characterized by stronger activity in the right hemisphere. This proposes an interesting opportunity to investigate further a neurophysiological framework for reading.

\section{References}

Badcock, N. A., Preece, K. A., de Wit, B., Glenn, K., Fieder, N., Thie, J., \& McArthur, G. (2015). Validation of the Emotiv EPOC EEG system for research quality auditory event-related potentials in children. PeerJ, 3, e907. doi: 10.7717/peerj.907

Bastos, A. M., Fries, P., Litvak, V., Moran, R., Friston, K.J., \& Bosman, C.A. (2015). A DCM study of spectral asymmetries in feedforward and feedback connections between visual areas V1 and V4 in the monkey. $\quad$ NeuroImage, 108, 460-475. doi: 10.1016/j.neuroimage.2014.12.081

Bosse, M.L., \& Valdois, S. (2009). Influence of the visual attention span on child reading performance: A cross sectional study. Journal of Research in Reading, 32(2), 230-253. doi: 10.1111/j.14679817.2008.01387.x

Castles, A., Coltheart, M., Larsen, L., Jones, P., Saunders, S., \& McArthur, G. (2009). Assessing the basic components of reading: Revision of the Castles and Coltheart test with new norms (CC2). Retrieved January 5, 2018, from www.motif.org.au

Clark, K.A., Helland, T., Specht, K., Narr, K.L., Manis, F.R., Toga, A.W., \& Hugdahl, K. (2014). Neuroanatomical precursors of dyslexia identified from pre -reading through to age 11. Brain, 137(12), 3136-3141.doi: 0.1093/brain/awu229

Coltheart, M., Rastle, K., Perry, C., Langdon, R., \& Ziegler, J. (2001). DRC: A dual route cascaded model of visual word recognition and reading aloud. Psychological Review, 108(1), 204-256. doi: 10.1037//0033-295X.108.1.204

Emotiv Systems, Inc., 2013. EMOTIV EPOC+ - 14 Channel Wireless EEG Headset. Retrieved from https://www.emotiv.com/epoc

González-Garrido, A.A., Gómez-Velázquez, F.R., \& Rodríguez-Santillán, E. (2014). Orthographic recognition in late adolescents: An assessment through event-related brain potentials. Clinical EEG and Neuroscience, 45(2), 113-121. doi: 10.1177/1550059413489975 
Gori, S., Cecchini, P., Bigoni, A., Molteni, M., \& Facoetti, A. (2014). Magnocellular-dorsal pathway and sub-lexical route in developmental dyslexia. Frontiers in Human Neuroscience, 8(460). doi: 10.3389/fnhum.2014.00460

Goswami, U., Power, A.J., Lallier, M., \& Facoetti, A. (2014). Oscillatory "temporal sampling" and developmental dyslexia: Toward an overarching theoretical framework. Frontiers in Human Neuroscience, 8(904). doi: 10.3389/fnhum.2014.00904

Jiménez-Bravo, M., Marrero, V., \& Benítez-Burraco, A. (2017). An oscillopathic approach to developmental dyslexia: From genes to speech processing. Behavioural Brain Research, 329(1), 84-95. doi: 10.1016/j.bbr.2017.03.048

Kamel, N., \& Saeed Malik, A. (Eds.). (2015). EEG/ERP analysis: Methods and applications. Boca Raton, FL: CRC Press.

Macquarie Online Test Interface (2009). CC2 instructions for tester. Retrieved from www.motif.org.au/testsession/cc2/

Magazzini, L., \& Singh, K. D. (2018). Spatial attention modulates visual gamma oscillations across the human ventral stream. NeuroImage, 166, 219-229. doi: 10.1016/j.neuroimage.2017.10.069

Martin, A., Kronbichler, M., \& Richlan, F. (2016). Dyslexic brain activation abnormalities in deep and shallow orthographies: A meta-analysis of 28 functional neuroimaging studies. Human Brain Mapping, 37(7). doi: 10.1002/hbm.23202

Paulesu, E., Danelli, L., \& Berlingeri, M. (2014). Reading the dyslexic brain: Multiple dysfunctional routes revealed by a new meta-analysis of PET and fMRI activation studies. Frontiers in Human Neuroscience, 9(830). doi: 10.3389/fnhum.2014.00830

Pina Rodrigues, A., Rebola, J., Jorge, H., Ribeiro, M.J., Pereira, M., van Asselen, M., \& Castelo Branco, M. (2017). Visual perception and reading: New clues to patterns of dysfunction across multiple visual channels in developmental dyslexia. Investigative Ophthalmology \& Visual Science, 58, 309-317. doi: 10.1167/iovs.16-20095

Raven, J., Raven, J. C., \& Court, J. H. (2003). Manual for Raven's Progressive Matrices and Vocabulary Scales. San Antonio, TX: Harcourt Assessment.

Richlan, F., Kronbichler, M., \& Wimmer, H. (2013). Structural abnormalities in the dyslexic brain: A meta-analysis of voxel-based morphometry studies. Human Brain Mapping, 34(11), 3055-3065. doi: 10.1002/hbm.22127

Richlan, F. (2014). Functional neuroanatomy of developmental dyslexia: The role of orthographic depth. Frontiersin Human Neuroscience, 8, 347. doi: 10.3389/fnhum.2014.00347

Shaul, S., Arzouan, Y., \& Goldstein, A. (2012). Brain activity while reading words and pseudo-words: Acomparison between dyslexic and fluent readers. International Journal of Psychophysiology, 84(3), 270-276. doi: 10.1016/j.ijpsycho.2012.03.005

Shaywitz, S. E., \& Shaywitz, B. A. (2008). Paying attention to reading: The neurobiology of reading and dyslexia. Development and Psychopathology, 20(4), 1329-1349. doi: $10.1017 / \mathrm{S} 0954579408000631$

Žarić, G., Fraga González, G., Tijms, J., van der Molen, M. W., Blomert, L., and Bonte, M. (2014). Reduced neural integration of letters and speech sounds in dyslexic children scales with individual differences in reading fluency. PLoS One 9:e110337. doi: 10.1371/journal.pone.0110337

Žarić, G., Correia, J.M., Fraga González, G., Tijms, J., van der Molen, M.W., Blomert, L., \& Bonte, M. (2017). Altered patterns of directed connectivity within the reading network of dyslexic children and their relation to reading dysfluency. Developmental Cognitive Neuroscience, 23, 1-13. doi: 10.1016/j.den.2016.11.003 